\title{
Amélioration des sols : les fonds injectés dans la craie parisienne
}

\author{
Soil improvement : \\ injected grounds in Parisian chalk
}

\author{
Louis PAREZ \\ Président Honoraire du CFMS ${ }^{*}$
}

Rev. Franç. Géotech. n 63, pp. 51.56 (avril 1993)

\section{Résumé}

La réalisation de constructions souterraines de plus en plus profondes (parkings, locaux techniques...) a posé, ces dernières années, d'importants problèmes dans la région parisienne, particulièrement dans les zones où la nappe phréatique baigne une craie sous-alluviale très perméable $\left(10^{-4}\right.$ à $\left.10^{-2} \mathrm{~m} / \mathrm{s}\right)$.

L'enceinte latérale de ces fouilles est alors réalisée par des parois moulées qui ne peuvent pas être ancrées dans une couche imperméable.

Pour éviter des débits d'exhaure importants, il est nécessaire de réaliser un fond injecté abaissant la perméabilité moyenne de la craie en dessous de $5.10^{-6} \mathrm{~m} / \mathrm{s}$.

La réalisation de tels fonds injectés est délicate et doit suivre impérativement un certain nombre de règles énumérées ci-après. Le moindre écart risque de se traduire par un accroissement important du débit de pompage dont la correction est bien plus coûteuse que l'économie espérée.

\section{Abstract}

In recent years, deeper and deeper underground constructions have been built (carparks, technical buildings,...) in the Paris area. This has raised important questions, particularly in the areas where there is a very permeable sub-alluvial chalk $\left(10^{-4}\right.$ to $\left.10^{-2} \mathrm{~m} / \mathrm{s}\right)$ under the ground water.

The surrounding diaphragm walls of these excavations cannot be anchored in a waterproof layer.

To avoid an important pumping flow, it is necessary to lower the average permeability of the chalk below $5 \cdot 10^{-6} \mathrm{~m} / \mathrm{s}$ by creating an injected ground.

The realisation of such injected grounds is very delicate and must absolutely follow a number of rules explained below. Any deviation from these rules could cause an important increase of the pumping rate which would cost more to correct than the changes could hope to save.

\footnotetext{
- 4. place de Mexico, 75116 Paris.
} 


\section{LE PROBLÈME POSÉ}

La réalisation de sous-sols de plus en plus profonds pour des parkings ou des locaux techniques pose des problèmes importants en région parisienne où la nappe est proche de la surface.

Lorsqu'il n'existe pas de couche géologique suffisamment imperméable à une profondeur permettant d'ancrer économiquement une paroi d'étanchéité périphérique il n'y a guère que deux solutions:

- ou bien exécuter un plancher inférieur étanche et capable de résister à la poussée de la nappe en crue, soit grâce aux seules charges permanentes de l'ouvrage, soit avec l'aide de tirants ou de pieux travaillant en traction. Mais encore faut-il être capable de rabattre provisoirement et économiquement la nappe pendant la durée des travaux;

- ou bien réaliser, à une profondeur donnée, et sur une certaine épaisseur, une zone de sol injecté dont la perméabilité moyenne sera telle que la quantité d'eau à pomper soit inférieure à un seuil économique fixé.

Cette dernière solution est relativement facile à réaliser dans le Lutétien (marnes et caillasses - calcaire grossier fissuré).

Elle est beaucoup plus délicate dans la craie sous-alluviale de la région parisienne où elle doit suivre impérativement un certain nombre de dispositions.
Le moindre écart risque de se traduire par un accroissement important du débit de pompage, dont la correction est bien plus coûteuse que l'économie espérée.

\section{LA CRAIE PARISIENNE, MATÉRIAU INJECTABLE}

Avec J. FLORENTIN, G. LHÉRITEAU et M. FAHRI, nous avons publié, au Congrès International de Paris en 1961, une étude de la craie en Bassin parisien.

Cette étude indique que la craie en place est un matériau hétérogène: mélange de blocs séparés par des zones plus marneuses.

Plus récemment, des essais de laboratoire réalisés, à Sol-Essais, sur des prélèvements obtenus dans les banlieues sud-ouest (Issy-les-Moulineaux, Boulogne) indiquent un indice de plasticité faible ( 3 à 8$)$ et des limites de liquidité peu variables avec la profondeur (26 à $28 \%$ ). Mais ce qui est le plus intéressant pour notre problème, c'est la granulométrie et la porosité des couches supérieures de la craie sous-alluviale.

En admettant, avec CAMBEFORT, que la limite de pénétrabilité d'un coulis argile-ciment se situe, sur la courbe granulométrique de la craie entre 0,7 et $1 \mathrm{~mm}$ de diamètre des grains, cette limite est dépassée par 45

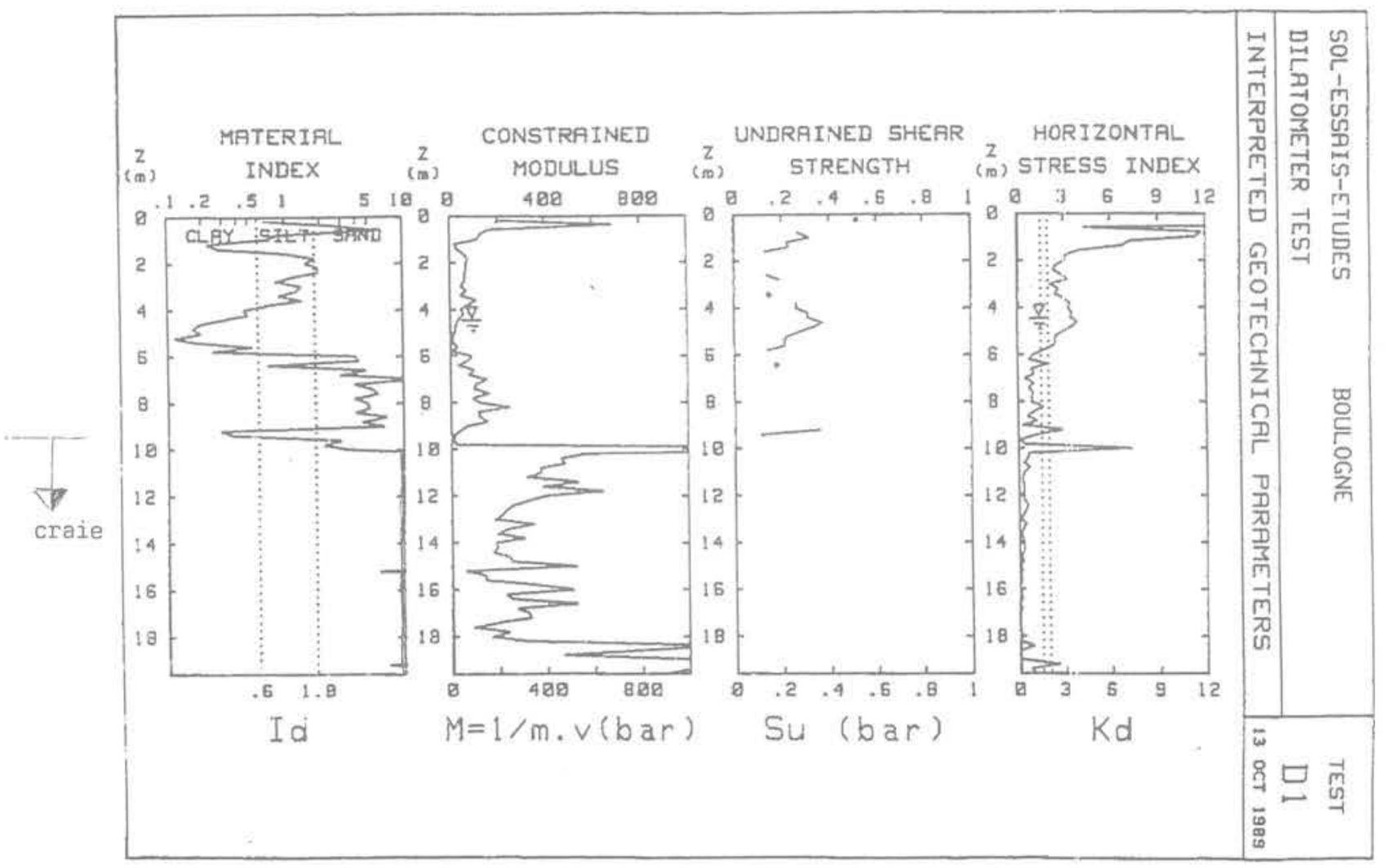

Fig. 1. - Essai au dilatomètre Marchetti dans la craie.

Fig. 1. - Marchetti Dilotometer Test in the chalk. 
à $70 \%$ des constituants de la craie, selon les échantil. lons.

Bien qu'il soit très difficile de carotter correctement la craie, on a pu y mesurer des porosités allant de 43 à $47 \%\left(\frac{\text { volume des vides }}{\text { volume total }}\right)$.

Ainsi, pour une porosité moyenne de $45 \%$, la quantité de coulis qui peut être injectée dans la craie de la région parisienne peut varier de 20 à $31 \%$ de son volume.
Evidemment, les parties les plus fines, très marneuses, ne sont pas injectables, mais elles sont aussi très imperméables $\left(\mathrm{k}=10^{-7}\right.$ à $\left.10^{-8} \mathrm{~m} / \mathrm{s}\right)$.

On voit donc que la craie qui nous intéresse se comporte, pour une bonne partie, comme un sable grossier injectable.

Les essais que nous y avons réalisés au dilatomètre Marchetti et au piézocône le confirment bien (u est souvent négatif).

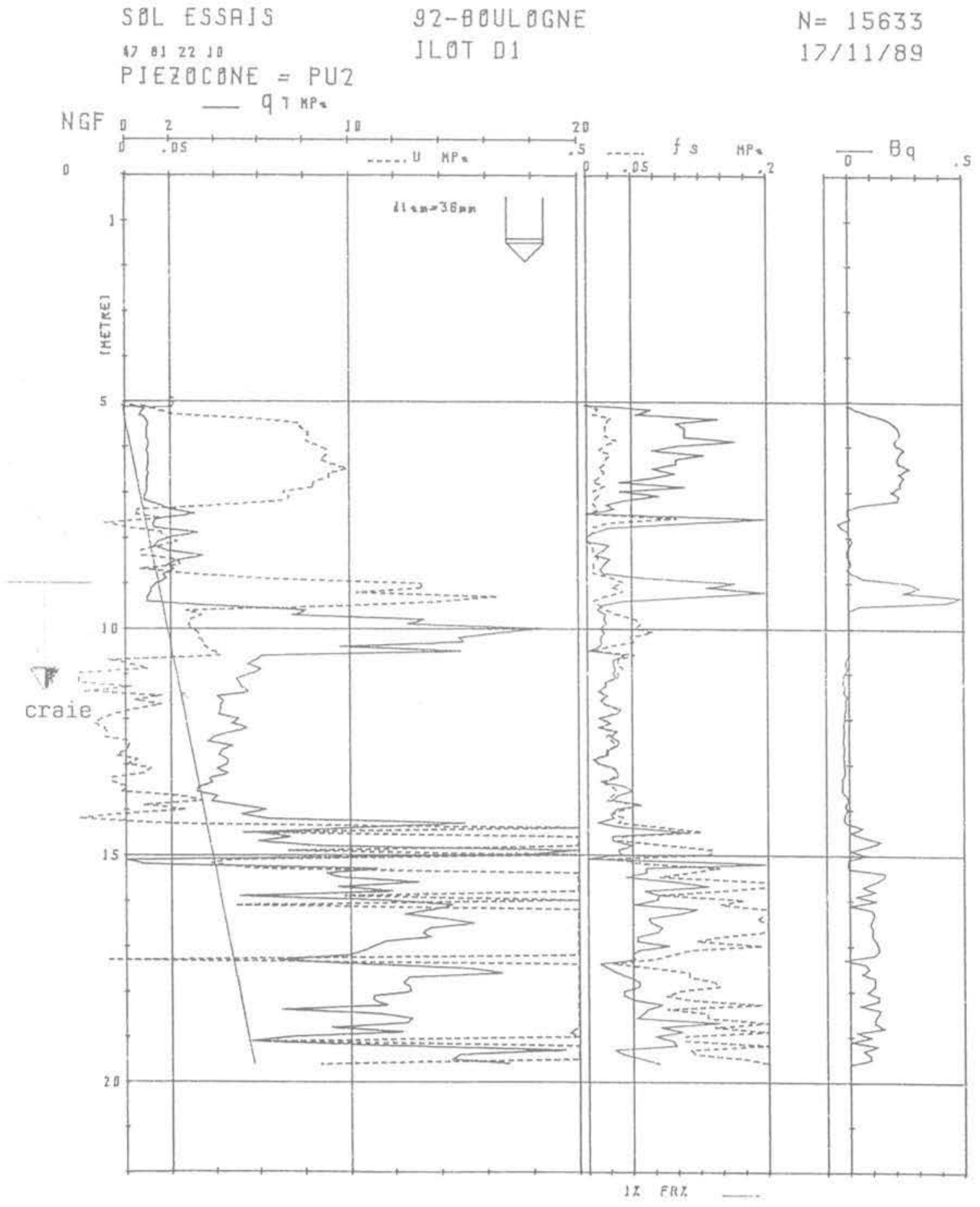

Fig. 2. - Essai piézocône dans la craie.

Fig. 2. CPTU in the chalk. 


\section{LA CRAIE PARISIENNE, MATÉRIAU TRĖS PERMÉABLE}

Tous les foreurs d'eau de la région ont su exploiter la nappe de la craie qui est très généreuse.

Les valeurs de perméabilité trouvées dans la craie par des essais de pompage sont souvent comprises entre $10^{-4}$ et $10^{-3} \mathrm{~m} / \mathrm{s}$. Elles peuvent parfois monter à $10^{-2} \mathrm{~m} / \mathrm{s}$. Ces chiffres sont semblables aux valeurs trouvées dans les alluvions anciennes.

Au piézocône on obtient couramment des $t_{50}$ de 5 à 2 secondes, ce qui correspond aux valeurs de perméabilité indiquées ci-dessus.

\section{LE PROJET DE FOND INJECTÉ}

\subsection{Epaisseur}

Le but est d'obtenir, pour un abaissement donné de la nappe, un débit de pompage acceptable économiquement.

Si l'enceinte périphérique est absolument étanche (pas de fuites à la paroi moulée) le débit $Q$ en $\mathrm{m}^{3} / \mathrm{h}$ à pomper, pour un abaissement $h$ de la nappe, dans une fouille de surface $S$ en $\mathrm{m}^{2}$ avec un fond injecté d'épaisseur e en $\mathrm{m}$ et de perméabilité moyenne $\mathrm{k}$ en $\mathrm{m} / \mathrm{s}$ est donné par

$$
\mathrm{Q}=3600 \cdot \frac{\mathrm{h}}{\mathrm{e}} \cdot \mathrm{k} \cdot \mathrm{S}
$$

En gros, avec un fond dont la perméabilité est $4.10^{-6} \mathrm{~m} / \mathrm{s}$ et l'épaisseur $3 \mathrm{~m}$, on pompe $1 \mathrm{~m}^{3} / \mathrm{h}$ par $100 \mathrm{~m}^{2}$ de surface et par $2 \mathrm{~m}$ de rabattement.

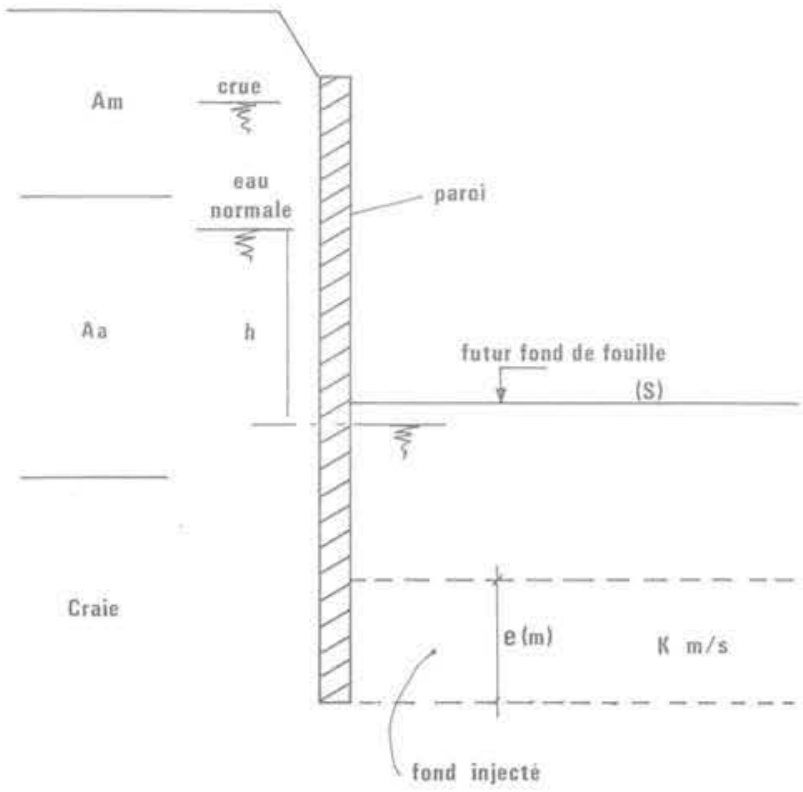

Fig. 3. - Coupe type.

Fig. 3. - Typical view.
Sont donnés :

- le rabattement en cote normale de la nappe,

- la surface de la fouille,

- le débit accepté par le maître d'ouvrage en nappe normale.

L'entrepreneur déterminera l'épaisseur du fond en fonction de la perrnéabilité moyenne qu'il espère obtenir. I ne négligera pas de tenir compte du débit de fuite de l'enceinte périphérique.

La puissance installée devra être fonction de la hauteur des crues, mais ce n'est pas cela qui influence beaucoup le coût annuel d'exploitation.

\subsection{Stabilité}

La paroi périphérique est vérifiée classiquement en tant que soutènement et à la condition de renard.

La stabilité du fond au soulèvement est calculée en supposant le niveau de l'eau à l'intérieur de la fouille proche de la cote de terrassement. Le poids volumique du sol déjaugé est pris égal à $10 \mathrm{kN} / \mathrm{m}^{3}$ et on admet par sécurité que la perte de charge se produit en partie haute du fond injecté.

La cote du dessus du fond injecté est alors vérifiée dans les cas suivants:

- en phase travaux avec la cote de crue admise en période de chantier;

- en phase définitive, avec la crue maximale, en prenant en compte le poids mort du bâtiment si celui-ci est réparti au-dessus du fond injecté;

- on conserve une garde d'au moins un mètre entre la base des alluvions anciennes et le dessus du fond injecté pour éviter une surconsommation de coulis.

Enfin il faut prévoir des puits de décharge, si le fond de fouille est peu perméable.

\section{RÉALISATION DU FOND INJECTÉ}

\subsection{Volume à injecter}

Comme indiqué précédemment, les quantités de coulis peuvent varier de 20 à $31 \%$ du volume du fond injecté. Comme il s'en perd toujours un peu en parties haute et basse, il faut compter sur un minimum de $25 \%$ avec un maximum de $35 \%$

\subsection{Maillage}

Les forages d'injection sont répartis selon un certain maillage dont l'entr'axe ne devrait pas dépasser $2,50 \mathrm{~m}$ en partie courante.

La première ligne, à proximité de la paroi périphérique, doit être au maximum à $1 \mathrm{~m}$ de celle-ci, l'entr'axe des forages étant de $2 \mathrm{~m}$.

La deuxième ligne, à $2 \mathrm{~m}$ de la précédente, aura le même entr'axe de $2 \mathrm{~m}$. 

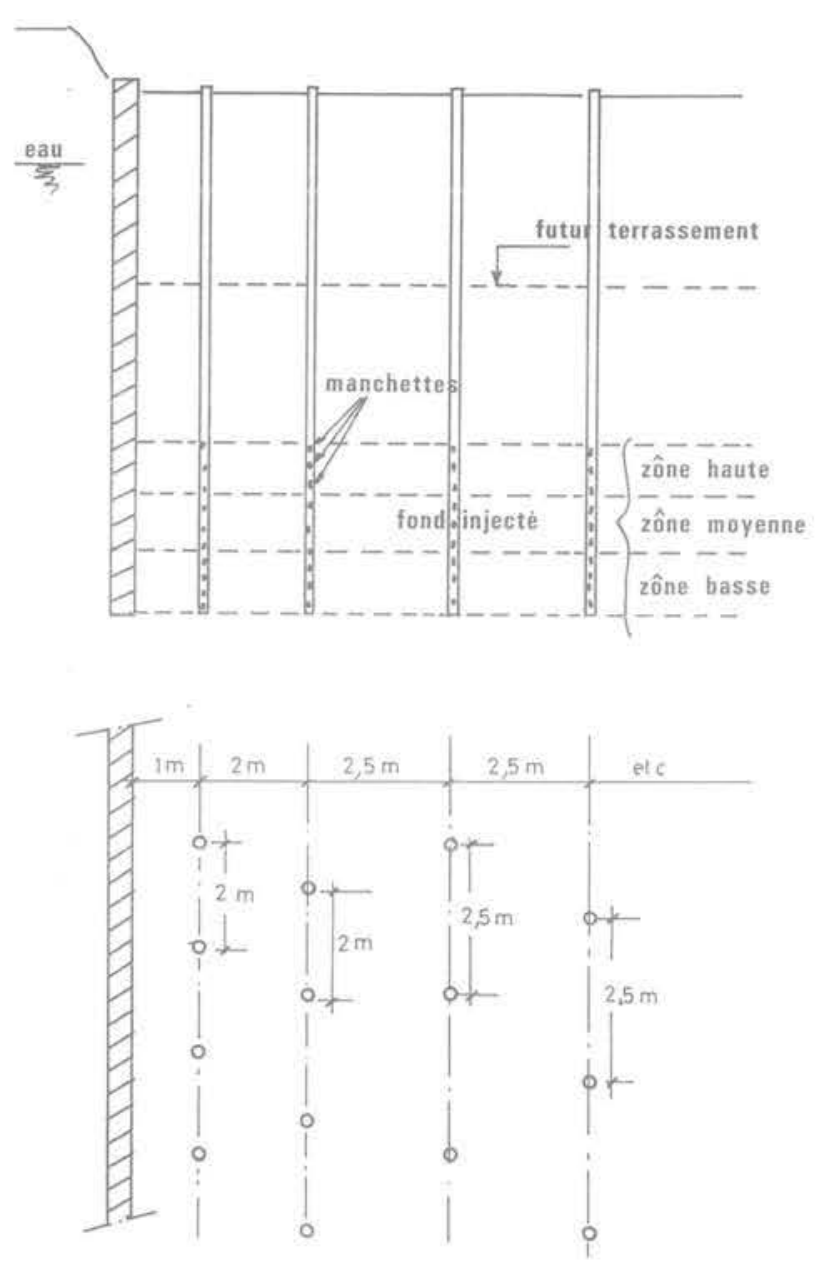

Fig. 4. - Implantation des forages.

Fig. 4. - Implantation of the boreholes.

\subsection{Tubes}

L'injection se fait par des tubes à manchettes scellés dans un coulis de gaine ( 3 manchettes par mètre dans la hauteur du fond injecté).

\subsection{Passes}

L'épaisseur du fond injecté est partagée en trois zones de hauteurs sensiblement égales.

L'injection se fait, manchette par manchette, à l'obturateur double et par passes:

- d'abord tous les forages sont injectés en zone haute sans trop pousser la pression, en notant bien les volumes injectés à chaque manchette;

- ensuite, tous les forages sont injectés en zone basse, dans les mêmes conditions;

- puis la zone centrale de chaque forage est injectée en augmentant la pression (en faisant attention au fait que les pertes de charge dans les tuyauteries peuvent être très importantes).
Le volume de coulis injecté à chaque manchette est toujours noté.

Aucun tube ne doit évidemment échapper.

Il est souvent nécessaire de reprendre la zone basse et la zone centrale.

En fin d'injection la montée en pression doit être significative et le volume injecté doit se placer dans la fourchette indiquée précédemment.

\section{POINTS DÉLICATS}

Le raccordement du fond injecté avec la paroi périphé rique est toujours délicat. Les bases de l'un et de l'autre devraient être au même niveau et chaque panneau de paroi devrait comporter au moins un tube par lequel on puisse faire, à la fin, une injection destinée à améliorer le raccordement.

De la même façon, la traversée du fond injecté par des pieux comporte un gros risque de fuites, même si le fond est injecté après l'exécution des pieux, car le coulis semble s'arrêter un peu avant la barrière que représente le béton des pieux... ou de la paroi.

\section{CONTRÔLE}

Avant de réaliser les terrassements, il faut vérifier le débit global de fuite par quelques puits de pompage dont le forage est arrêté nettement au-dessus du fond injecté.

De même, quelques piézomètres sont installés à l'intérieur de l'enceinte et au moins un à l'extérieur.

Si le débit global est supérieur au débit contractuel l'injection est reprise.

Pour ces essais de pompage, la méthode rapide donnée par Madame IAGOLNITZER et Monsieur MONNET de l'Entreprise BACHY dans le n 55 de la RFG d'avril 1991 est tout à fait recommandée.

Il est, en général, difficile d'apprécier le débit qui peut passer à travers la paroi périphérique (entre les panneaux ou par une zone de bétonnage défectueux).

\section{CONCLUSION}

En appliquant les indications précédentes, on doit obtenir une perméabilité moyenne du fond de 3 à $4.10^{-6}$ $\mathrm{m} / \mathrm{s}$ dans la craie parisienne.

Avec une équipe très minutieuse, et en injectant deux fois les zones basse et médiane, on peut arriver à ob- 
tenir $2.10^{-6}$ qui semble actuellement la limite écono. mique.

Etant donné l'hétérogénéité de la craie, le maître d'ouvrage ne devrait pas imposer une valeur de débit global mais plutôt une fourchette de valeurs (par exemple $\mathrm{D} \pm 20 \%$ )

On pourrait aussi envisager d'intéresser l'entrepreneur à l'économie de pompage.

\section{BIBLIOGRAPHIE}

FLORENTIN J, et al. (1961), Observations faites sur la craie comme couche de fondation. $5^{\circ}$ Congrès International de Mécanique des Sols, Paris, 1/17, pp. 101-105.

IAGOLNITZER Y. et MONNET A. (1991), Interprétation d'essai de pompage dynamique dans les en. ceintes fermées. Revue Française de Géotechnique $\mathrm{n}^{\circ} 55$, avril 1991, pp. 34-35. 